\title{
On the multiscale origins of fracture resistance in human bone and its biological degradation
}

\section{DISCLAIMER}

This document was prepared as an account of work sponsored by the United States Government. While this document is believed to contain correct information, neither the United States Government nor any agency thereof, nor the Regents of the University of California, nor any of their employees, makes any warranty, express or implied, or assumes any legal responsibility for the accuracy, completeness, or usefulness of any information, apparatus, product, or process disclosed, or represents that its use would not infringe privately owned rights. Reference herein to any specific commercial product, process, or service by its trade name, trademark, manufacturer, or otherwise, does not necessarily constitute or imply its endorsement, recommendation, or favoring by the United States Government or any agency thereof, or the Regents of the University of California. The views and opinions of authors expressed herein do not necessarily state or reflect those of the United States Government or any agency thereof or the Regents of the University of California. 


\title{
On the multiscale origins of fracture resistance in human bone and its biological degradation
}

\author{
E. A. Zimmermann ${ }^{\mathrm{a}}$, H. D. Barth ${ }^{\mathrm{a}, \mathrm{b}}$, and R. O. Ritchie $\mathrm{e}^{\mathrm{a}, \mathrm{e}^{*}}$ \\ aMaterials Sciences Division, Lawrence Berkeley National Laboratory, Berkeley, CA, USA \\ bExperimental Systems Group, Lawrence Berkeley National Laboratory, Berkeley, CA, USA \\ cDepartment of Materials Science \& Engineering, University of California, Berkeley, CA, USA
}

\begin{abstract}
Akin to other mineralized tissues, human cortical bone can resist deformation and fracture due to the nature of its hierarchical structure, which spans the molecular to macroscopic length-scales. Deformation at the smallest scales, mainly through the composite action of the mineral and collagen, contributes to bone's strength or intrinsic fracture resistance, while crack-tip shielding mechanisms active on the microstructural scale contribute to the extrinsic fracture resistance once cracking begins. The efficiency with which these structural features can resist fracture at both small and large length-scales becomes severely degraded with such factors as aging, irradiation and disease. Indeed aging and irradiation can cause changes to the cross-link profile at fibrillar length-scales as well as changes at the three orders of magnitude larger scale of the osteonal structures, both of which combine to inhibit the bone's overall resistance to the initiation and growth of cracks.
\end{abstract}

\footnotetext{
* To whom correspondence should be addressed. E-mail address: roritchie@lbl.gov.
} 


\section{Introduction}

Human cortical bone develops its unique mechanical properties of strength, stiffness, and toughness through its complex architecture. In healthy bone, the structure, which evolves over multiple length-scales, is able to resist fracture through a combination of intrinsic mechanisms acting at small length-scales to promote plasticity and extrinsic mechanisms acting at the microstructural level to shield the extension of a growing crack. However, many biological factors, such as aging, disease, or environmental influences, can change this structure and in turn affect the bone's ability to efficiently absorb energy. Recent results, specifically for aging and irradiation, demonstrate how changes in bone's architectural structure at small and large length-scales can inhibit the toughening mechanisms that are active in healthy bone and consequently degrade its mechanical properties, leading to increased fracture risk.

Aging is a primary concern to the medical community, where the deterioration in bone quality is understood to increase the risk of fracture in the aging population $(1,2)$. Indeed, human cortical bone is known to lose plasticity and toughness as it ages $(3,4)$. An understanding of the structural changes occurring over various length-scales due to such biological aging and their consequent effect on the mechanical properties is thus critical (5).

Irradiation also has deleterious consequences to the macroscopic mechanical properties of cortical bone (6). The effects of radiation are of primary concern for the sterilization of bone allografts (7), but also for the research community, who often use high-flux synchrotron radiation while analyzing the mechanical properties of bone in situ $(8,9)$. The latter would provide misleading results if the radiation dose used in the experiments damages the bone matrix. Indeed, radiation alters the bone structure at its smallest length-scales, which has 
deleterious consequences to its resistance to the initiation and growth of cracks $(6,10)$.

Here, we review the structure of healthy human cortical bone and its role in resisting fracture. We further utilize a hierarchical perspective to describe the development of toughness over multiple length-scales and apply this specifically to the deterioration in bone matrix properties with aging and irradiation.

\section{Structure of human cortical bone}

Two features of bone's hierarchical structure that are especially influential to its mechanical properties are the mineralized collagen fibril at sub-micron levels and the secondary osteon at the microstructural level. The collagen fibril is the basic building block of many biological materials (from tendons and skin to hard mineralized tissues, such as bone) and is composed of a periodic array of collagen molecules impregnated with mineral with a 67-nm stagger (11). The mineral consists of nanocrystals of hydroxyapatite (HA) deposited within this framework, specifically first within the gaps zones (between the heads and tails of the collagen molecules) $(12,13)$ and later in the overlap zones as well as the surface of the fibril (14-16).

Cross-links within and between the fibrils play a large role in stabilizing this periodic structure. In human cortical bone, cross-links occur in two forms: i) enzymatic cross-links and non-enzymatic cross-links. Immature enzymatic crosslinks (DHLNL and HLNL ${ }^{1}$ ) occur intrafibrillarly between collagen at specific sites along the molecule and are thought to mature into a more stable interfibrillar cross-link (i.e., pyridinoline and pyrrole). Likewise, non-enzymatic cross-links (also known as advanced glycation end-products (AGEs), such as

${ }^{1}$ DHLNL and HLNL are, respectively, dehydrodihydroxynorleucine and dehydrohydroxylysinonorleucine. 
pentosidine) occur via a glucose-mediated reaction to form both intra- and interfibrillar links along the collagen backbone (17). While the enzymatic crosslink profile stabilizes around 10-15 years of age $(18,19)$, AGEs can increase up to five-fold with age (19-21).

At higher length-scales, the secondary osteon is the foremost feature associated with fracture resistance. The osteon has a central vascular cavity ( $\sim 90$ $\mu \mathrm{m}$ in diameter) that is concentrically surrounded by $5-\mu \mathrm{m}$-thick sheets of lamellae (22). At its outer boundary, the secondary osteon contains an interface, called the cement line, which represents an especially weak region due to its hyper-mineralization; it thus acts as a site where major microcracks form and its alignment with the long axis of the long bones is the primary cause of the anisotropic toughness of cortical bone when tested perpendicular and parallel to the osteons. It is through this hierarchical structure that human cortical bone is able to develop its unique combination of strength and toughness.

\section{Toughening mechanisms in cortical bone}

Healthy human cortical bone resists fracture through mechanisms built into the many levels of its unique structure. This resistance can be conveniently separated into those intrinsic mechanisms that promote plasticity at small lengthscales and extrinsic mechanisms that shield the growing crack at larger lengthscales (Fig. 1). The relative potency of these mechanisms between diseased and healthy bone is apparent by measuring the bone's strength and fracture toughness, specifically through the crack-growth resistance curve. ${ }^{2}$

\footnotetext{
2 The crack resistance-curve or R-curve provides an assessment of the fracture toughness in the presence of subcritical crack growth. It involves measurements of the crack-driving force, e.g., the stress intensity $K$ or $J$ integral, as a function of crack extension $(\Delta a)$. The value of the driving force at $\Delta a \rightarrow 0$ provides a measure of the crack-initiation toughness whereas the slope (used in this study) and/or the maximum value of the Rcurve can be used to characterize the crack-growth toughness. Indeed, the slope of a rising R-curve is directly related to the potency of the extrinsic toughening mechanism involved.
} 
The intrinsic mechanisms of toughness in bone represent the inherent resistance of the material to deformation and fracture; they essentially result from plasticity. These fundamental characteristics of the bone can be quantified in terms of the strength and ductility of the bone during tension or compression testing, as well as through the initiation toughness of the R-curve (i.e., the toughness as $\Delta a \rightarrow 0$ ). These macroscopic measurements give a sense of the bone's intrinsic ability to resist fracture, but the plasticity originates from mechanisms at work at the smallest length-scales. Intrinsic toughening in bone includes deformation of the mineral and collagen components (e.g., molecular uncoiling). However, as bone acquires its unique combination of strength and toughness from both the mineral's strength and the matrix's ductility, the major contributions to the intrinsic toughness are derived from deformation of the composite, i.e., the mineralized collagen fibril. As load is applied in the elastic regime, the load is transmitted to the smallest components and carried as tension in the mineral platelets transferred via shearing of the collagenous matrix (23). Once yielding occurs, the mineralized fibril and the fibril matrix begin to decouple (24); this mechanism termed fibrillar sliding (Fig. 1) is essential to promote plasticity at this length-scale $(25,26)$. Many aspects of the fibril's structure play a role in its ability to efficiently promote fibrillar sliding, such as the HA/collagen interface (27), intermolecular cross-linking (17) and sacrificial bonding (28). These fibrillar features constrain molecular stretching and provide the basis for the increased apparent strength of the collagen molecules without catastrophic failure of either component. This composite behavior of the protein and mineral phases enables a large regime of dissipative deformation once plastic yielding begins in mineralized tissues $(24,25,29)$ and other biological materials (30). This model of load transfer represents the principal plasticity mechanism at this length-scale. As in most materials, plasticity provides a major 
contribution to the intrinsic toughness by dissipating energy and forming "plastic zones" surrounding incipient cracks that further serve to blunt crack tips, thereby reducing the driving force (i.e., stress intensity) for crack propagation.

An even larger contribution to the fracture resistance of bone, however, arises at coarser length-scales in the range of 10 to 100s microns from mechanisms of extrinsic toughening. The extrinsic toughness can be quantified through the slope of the R-curve, i.e., the material's resistance to crack extension. Specifically, once the crack begins to grow, mechanisms are activated within the microstructure which inhibit further crack growth. Indeed, the primary driver for these mechanisms is the nature of the crack path and its interaction with the bonematrix structure. Two salient toughening mechanisms can be identified $(31,32)$ : uncracked ligament bridging and crack defection/twist (Fig. 1). Crack bridging occurs as microcracks form ahead of the crack tip, primarily along the hypermineralized interfaces at the bounds of the osteons, producing so called "uncracked-ligament" bridges. Specifically, the microcracks result in intact regions spanning the crack wake, which carry load that would otherwise be used to further extend the crack. Deflection of cracks is also a major toughening mechanism in human cortical bone, and is particularly potent in the transverse orientation when the crack is oriented perpendicular to the osteon. As the crack begins to grow, structural features (e.g., osteocyte lacunae and porosity) can deflect the crack path, but it is the largest features, specifically the secondary osteons and their brittle cement lines, that are the most effective. Crack deflection toughens normal bone by deviating the crack path from the plane of maximum tensile stress; in this way, the stress intensity at the crack tip decreases (typically by a factor of two or more) and requires a larger applied force to further 
propagate the crack. The resulting highly torturous crack path is a clear sign of this mechanism's significant contribution to the toughness. Thus, the key feature to the extrinsic toughness is the bone's ability to microcrack primarily at the hyper-mineralized osteonal boundaries (i.e., cement lines) leading to the toughening mechanisms of crack deflection and uncracked ligament bridging.

Through this complementary deformation at small length-scales mainly through fibrillar sliding (i.e., intrinsic toughening) as well as crack-tip shielding mechanisms at larger length-scales (i.e., extrinsic toughening), healthy human cortical bone is able to derive its mechanical properties of strength and toughness over multiple dimensions.

\section{Aging-related structural changes}

In human cortical bone, aging results in changes to the bone structure over a range of length-scales that deteriorate its quality and increase the risk of fracture. A convenient way to assess this deterioration in quality is by analyzing the effectiveness of the intrinsic and extrinsic toughening mechanisms with age.

The bone's macroscopic mechanical properties of strength and toughness (Fig. 2a) are clearly degraded with age. Specifically, in comparison to Young bone, the losses in strength (through a reduction in peak strength and ductility) and crack initiation toughness with age indicate deterioration in intrinsic toughness (5). A convenient experiment to further probe the smallest lengthscales, where the intrinsic toughness develops, is mechanical testing with simultaneous synchrotron in situ small-angle $\mathrm{x}$-ray scattering and wide-angle $\mathrm{x}$ ray diffraction (SAXS/WAXD) measurements (Fig. 3), which provide measurements of fibrillar-level deformation. The SAXS/WAXD experiments indicate that for a given strain applied to the bone tissue, the strain carried by the 
mineralized collagen fibrils is significantly less, by some $25 \%$, in Aged, as compared to Young, bone (Fig. 4a,b). This implies that in Aged bone, stress and strain are not efficiently transferred to the smallest length-scales as in Young bone; the deformation of Aged fibrils is constrained and higher levels of the hierarchy must contribute more to the mechanism of deformation (5).

This notion of a lack of plasticity in Aged fibrils is completely consistent with cross-linking measurements on the same set of bones (Fig. 4c), and elsewhere (19, 20) which demonstrates that aging results in increased AGEs cross-links. The post-translational formation of non-enzymatic AGEs cross-links occurs via a glucose-mediated reaction, where free amino groups in the collagen molecules form molecular cross-links (17). An elevated concentration of AGEs is known to occur with aging, certain diseases and specific therapies; the underlying biological changes typically associated with increased AGEs formation are altered glycation, low turnover, and increased oxidative reactions (33). In terms of mechanical properties, the cross-links act to degrade the structural integrity of the fibrils by stiffening them to restrict fibrillar sliding (plasticity) and consequently degrade the ductility, strength and intrinsic toughness of the bone. Further, our results are consistent with analytical models of fibril deformation (34) which predict an inhibition of fibrillar sliding with non-enzymatic crosslinking, and with experiments (35) showing a reduced toughness in bone glycated in vitro. Taken as a whole, the evidence at submicron length-scales strongly suggests that aging results in changes to the collagen environment, which specifically act to constrain the fibril's capacity to deform. The loss in plasticity at these nanometer length-scales directly degrades the intrinsic toughness, thereby contributing to the increased fracture risk. 
A corresponding decrease in crack-growth toughness (i.e., the slope of the Rcurve) has also been seen with aging $(4,5)$, indicating a change in potency of the extrinsic toughening mechanisms. In the longitudinal orientation, microcracks largely form parallel or ahead of the main growing crack and result in the formation of uncracked-ligament bridges (Fig. 5a). Detailed 2-D environmental scanning electron microscopy studies show that in general the size and frequency of these crack bridges is much lower in Aged bone (Fig. 5b). We believe that this is the result of an increased density of osteons (Fig. 5c,d) (4), which is characteristic of Aged bone due to a higher rate of turnover. As the nominal size of the uncracked ligaments scales with the 3-D osteonal spacing, the increased osteonal density leads to smaller and less frequent crack bridges. The marked reduction in the slope of the fracture toughness R-curves with age (Fig. 2a) is completely consistent with this diminished contribution from extrinsic toughening by crack bridging.

This deterioration in mechanical properties of human cortical bone with age has a direct relation to the suppression of toughening mechanisms caused by biological aging of the structure. Aging leads to a suppression of fibrillar sliding associated with increased collagen cross-linking at molecular levels, as well as smaller crack bridges due to the higher osteon density, which decreases the resistance to crack extension.

\section{Radiation-related structural changes}

Akin to the effects of aging, x-ray irradiation exposure can lead to an analogous yet more dramatic deterioration in cortical bone's overall resistance to fracture (Fig. 2b). Macroscopic mechanical tests clearly indicate that a primary

aspect of the irradiation-induced loss in fracture resistance can be attributed to a 
complete loss in post-yielding (plastic) deformation (intrinsic toughness) for radiation exposures of $70 \mathrm{kGy}^{3}$ and above (6). Complementary, SAXS/WAXD analyses indicate a loss in fibril strain and a significant decrease in fibrillar sliding (10). Specifically, at the onset of plastic deformation in healthy (unirradiated) bone (Figs. 6a), the strain in the mineral becomes roughly constant with increasing tissue strain. In contrast, once plastic deformation begins in the irradiated bone, the tissue fails due to degradation of the collagen matrix, which acts to totally eliminate the bone's capacity for plastic deformation; in materials science terms, the bone simply "embrittles" due to irradiation.

Analogous to aging in bone, this loss in intrinsic resistance to fracture following radiation exposure occurs due to changes in the cross-link profile (3639) which acts to suppress the prevailing plasticity mechanism in bone of fibrillar sliding. In this regard, the profile and distribution of collagen cross-links have a major influence on bone function.

The enzymatic cross-link profile was investigated via a Fourier transform infrared (FTIR) analysis on unirradiated and 70-kGy irradiated bone; the ratio of mature (e.g., Pyr) to immature (e.g., DHNLN) cross-links can be determined by comparing the peak areas of the 1660 and $1690 \mathrm{~cm}^{-1}$ sub-bands in the amide I band (1585 - $\left.1720 \mathrm{~cm}^{-1}\right)$, indicating the relative proportion of enzymatic crosslinks (40). For bone exposed to $70 \mathrm{kGy}$ of irradiation (10), the ratio of 1660:1690 peak areas decreased by almost two-thirds (Fig. 6b). The decrease in this ratio with irradiation exposure suggests a greater proportion of immature cross-links to mature cross-links following irradiation (40); indeed, either an increase in immature cross-links or a reduction in mature cross-links could disrupt the

\footnotetext{
${ }^{3}$ A Gray (Gy) is a unit of adsorbed dosage of ionizing radiation, equivalent to absorbed energy per unit mass $(1 \mathrm{~Gy} \equiv 1 \mathrm{~J} / \mathrm{kg})$. At the low dose end, the average radiation from an abdominal x-ray is $\sim 1.4 \mathrm{mGy}$; that from a pelvic CT scan is $25 \mathrm{mGy}$. At the high dose end ( $>10 \mathrm{kGy}$ ), gamma irradiation is commonly used to terminally sterilize allograft tissues and bones.
} 
integrity of the mature cross-links, such as covalent hydroxypyridinium, leading to premature failure of the tissue

Finally, we also find an increase in the concentration of the non-enzymatic (AGE) cross-links (Fig. 6c), which are known to suppress plasticity in older bone $(5,35)$. Indeed, we suspect that it is these cross-links that are primarily responsible for the restriction in bone plasticity from fibrillar sliding in irradiated bone. However, the mechanism of the apparent increased concentration of AGEs in irradiated bone is not known, but it may be possible that irradiation accelerates the formation of AGEs. Regardless of the mechanism, however, the increase in AGEs content is consistent with the inhibition of fibrillar sliding and loss of plasticity and hence intrinsic toughening, as also observed in aging bone. These harmful effects of bone irradiation can be observed at multiple lengthscales through the suppression of nano- and micro-scale plasticity mechanisms, and may be the result of alterations in the profile and distributions of the collagen cross-linking.

The extrinsic fracture resistance of cortical bone can also be affected by irradiation, as seen from the dramatic decline in the slope of the R-curve (Fig. $2 b)$. Exposure to $\mathrm{x}$-rays at dosages in excess of $70 \mathrm{kGy}$ can lead to radical changes in the crack path (6), with deflections of a far smaller magnitude (although more frequent) resulting in minimal crack-path deviations and an essentially linear crack trajectory (Fig. 7). It is the absence of such highly tortuous crack paths in irradiation-damaged bone, with minimal deviations from the plane of maximum tensile stress, which serves to curtail the high extrinsic toughness, which is characteristic of healthy bone in the transverse orientation.

\section{Summary}


The hierarchical structure of human cortical bone is specifically tailored to resist fracture at multiple length-scales, in particular through intrinsic mechanisms which provide for plasticity in bone acting at small, sub-micron, length-scales, as well as extrinsic mechanisms at larger, microscopic, lengthscales which act to "shield", and hence inhibit, the growth of cracks. Indeed, changes to the structure at one or more length-scales can severely curtail the effectiveness of these mechanisms leading to a degradation in the overall mechanical properties. Specifically, changes to the cross-link profile due to aging and radiation exposure play a significant role in limiting fibrillar sliding (i.e., plasticity) at the molecular level, whereas increases in the osteon density can have correspondingly negative repercussions to the crack-growth resistance.

Acknowledgements: This work was supported by the National Institute of Health (NIH/NIDCR) under grant no. 5R01 DE015633. We acknowledge the use of the two x-ray synchrotron beam lines 7.3.3 (SAXS/WAXD) and 8.3.2 (micro-tomography) at the Advanced Light Source at the Lawrence Berkeley National Laboratory (LBNL), which is supported by the Office of Science of the U.S. Department of Energy under contract no. DE-AC02-05CH11231. The authors wish to thank Dr. Tony Tomsia for his support, Drs. Maximilien Launey, Joel Ager, Hrishikesh Bale, Eric Schaible, and Alastair MacDowell at LBNL for their considerable experimental contributions, Prof. Tamara Alliston and Simon Tang at UCSF for performing the AGEs cross-link measurements, and Prof. Tony Keaveny and Mike Jekir at UC Berkeley for allowing us to use their facilities to machine bone samples.

\section{References}

1. D.B. Burr, Journal of Musculoskeletal \& Neuronal Interactions, 4 (2) (2004), pp. 184-186.

2. $\quad$ S.L. Hui, et al., Journal of Clinical Investigation, 81 (6) (1988), pp. 18041809.

3. R.W. McCalden, et al., Journal of Bone and Joint Surgery-American Volume, 75A (8) (1993), pp. 1193-1205.

4. R.K. Nalla, et al., Materials Science \& Engineering C, 26 (8) (2006), pp. 1251-1260.

5. E.A. Zimmermann, et al., Proceedings of the National Academy of Sciences, 108 (35) (2011), pp. 14416-14421. 
6. H.D. Barth, et al., Bone, 46 (6) (2010), pp. 1475-1485.

7. C.T. Vangsness, et al., American Journal of Sports Medicine, 31 (3) (2003), pp. $474-481$.

8. J.D. Almer and S.R. Stock, Journal of Structural Biology, 152 (1) (2005), pp. 14-27.

9. R. Voide, et al., Bone, 45 (2) (2009), pp. 164-173.

10. H.D. Barth, et al., Biomaterials, 32 (34) (2011), pp. 8892-8904.

11. A.J. Hodge and J.A. Petruska. Aspects of Protein Structure. Ed: G.N. Ramachandran. (New York: Academic Press, 1963).

12. W. Traub, et al., Proceedings of the National Academy of Sciences, 86 (24) (1989), pp. 9822-9826.

13. S. Weiner and W. Traub, Febs Letters, 206 (2) (1986), pp. 262-266.

14. A.L. Arsenault, Calcified Tissue International, 48 (1) (1991), pp. 56-62.

15. W.J. Landis, et al., Journal of Structural Biology, 117 (1) (1996), pp. 24-35.

16. M.E. Maitland and A.L. Arsenault, Calcified Tissue International, 48 (5) (1991), pp. 341-352.

17. A.J. Bailey, Mechanisms of Ageing and Development, 122 (7) (2001), pp. 735-755.

18. D.R. Eyre, et al., Biochemical Journal, 252 (2) (1988), pp. 495-500.

19. M. Saito, et al., Analytical Biochemistry, 253 (1) (1997), pp. 26-32.

20. D.R. Sell and V.M. Monnier, Journal of Biological Chemistry, 264 (36) (1989), pp. 21597-21602.

21. P. Odetti, et al., Annals of the New York Academy of Sciences, 1043 (1) (2005), pp. 710-717.

22. R.B. Martin and D.B. Burr. Structue, Function, and Adaptation of Compact Bone. (New York, NY: Raven Press, 1989).

23. I. Jager and P. Fratzl, Biophysical Journal, 79 (4) (2000), pp. 1737-1746.

24. H.S. Gupta, et al., International Journal of Fracture, 139 (3-4) (2006), pp. 425-436.

25. H.S. Gupta, et al., Proceedings of the National Academy of Sciences 103 (47) (2006), pp. 17741-17746.

26. H.S. Gupta, et al., Nano Letters, 5 (10) (2005), pp. 2108-2111.

27. B.H. Ji and H.J. Gao, Journal of the Mechanics and Physics of Solids, 52 (9) (2004), pp. 1963-1990.

28. G.E. Fantner, et al., Nature Materials, 4 (8) (2005), pp. 612-616.

29. S. Krauss, et al., Bone, 44 (6) (2009), pp. 1105-1110.

30. M.J. Harrington, et al., Journal of Structural Biology, 167 (1) (2009), pp. 4754.

31. K.J. Koester, et al., Nature Materials, 7 (8) (2008), pp. 672-677.

32. R.K. Nalla, et al., Biomaterials, 26 (2) (2005), pp. 217-231. 
33. M. Saito and K. Marumo, Osteoporosis International, 21 (2) (2010), pp. 195-214.

34. T. Siegmund, et al., Journal of Biomechanics, 41 (7) (2008), pp. 1427-1435.

35. D. Vashishth, et al., Bone, 28 (2) (2001), pp. 195-201.

36. A.J. Bailey, et al., Calcified Tissue International, 65 (3) (1999), pp. 203-210.

37. X. Banse, et al., Journal of Bone and Mineral Research, 17 (9) (2002), pp. 1621-1628.

38. S. Viguet-Carrin, et al., Osteoporos International, 17 (3) (2006), pp. 319-36.

39. X. Wang, et al., Bone, 31 (1) (2002), pp. 1-7.

40. E.P. Paschalis, et al., Journal of Bone and Mineral Research, 16 (10) (2001), pp. 1821-1828.

41. M.E. Launey, et al., Annual Review of Materials Research, 40 (2010), pp. 25-53. 

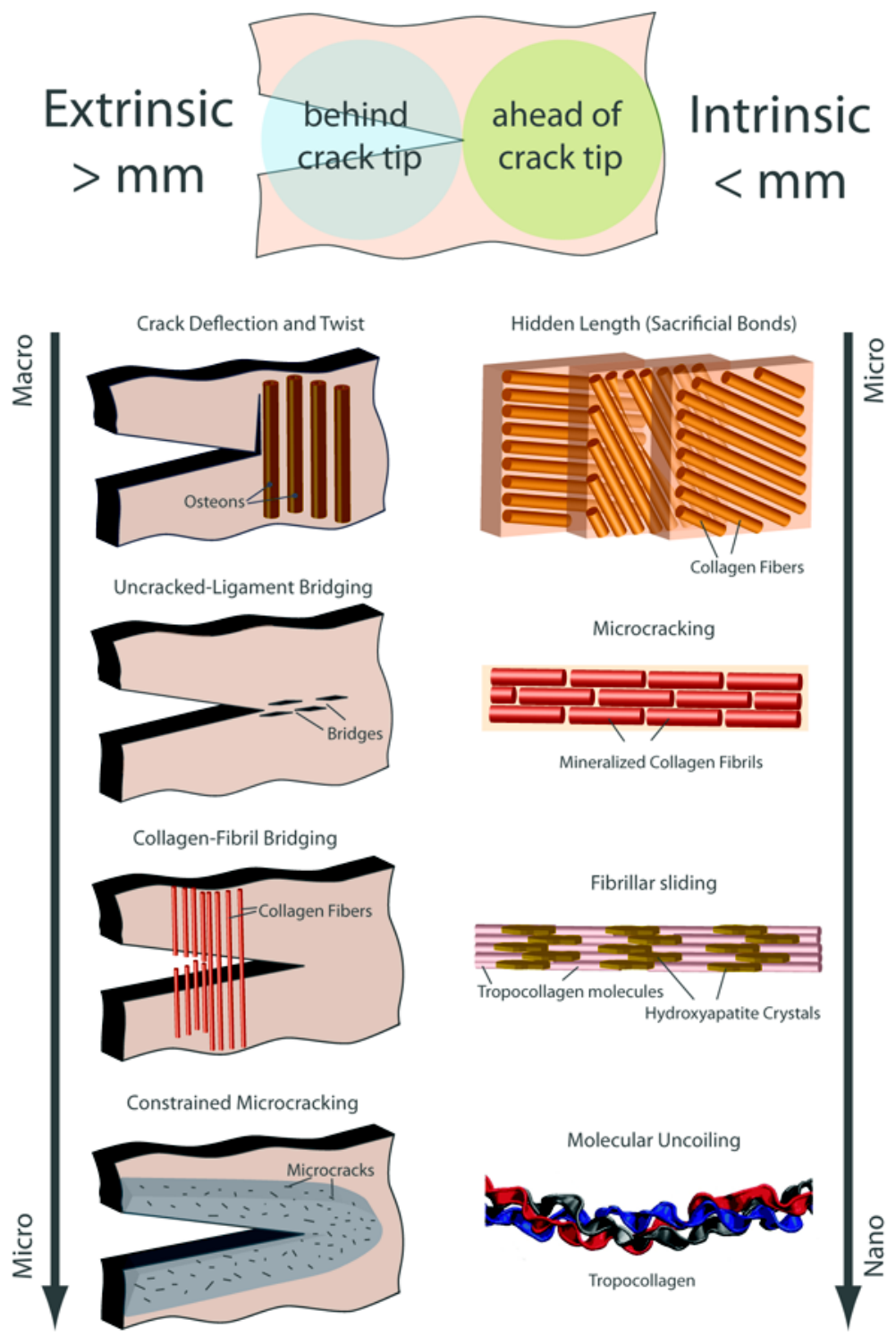

Fig. 1: The toughness of human cortical bone is derived through its hierarchical structure, from the nano- to macro-scale. Intrinsic mechanisms promoting plasticity, occur ahead of the crack tip, and are derived primarily at the smallest length-scales. In addition to contributions from molecular uncoiling, microcracking, and sacrificial bonding, fibrillar sliding has the largest impact on the inherent resistance of the HA/collagen composite. At higher length-scales, extrinsic mechanisms, such as uncracked ligament bridging and crack deflection, occur once the crack begins to grow and shield the crack tip from the full stress intensity. In this way, healthy human cortical bone resists fracture through complementary intrinsic and extrinsic contributions throughout its hierarchical structure. Aging, irradiation, and disease affect the efficiency with which these mechanisms can act by changing the structure and consequently degrade the toughness. This figure was adapted from Launey et al. (41). 

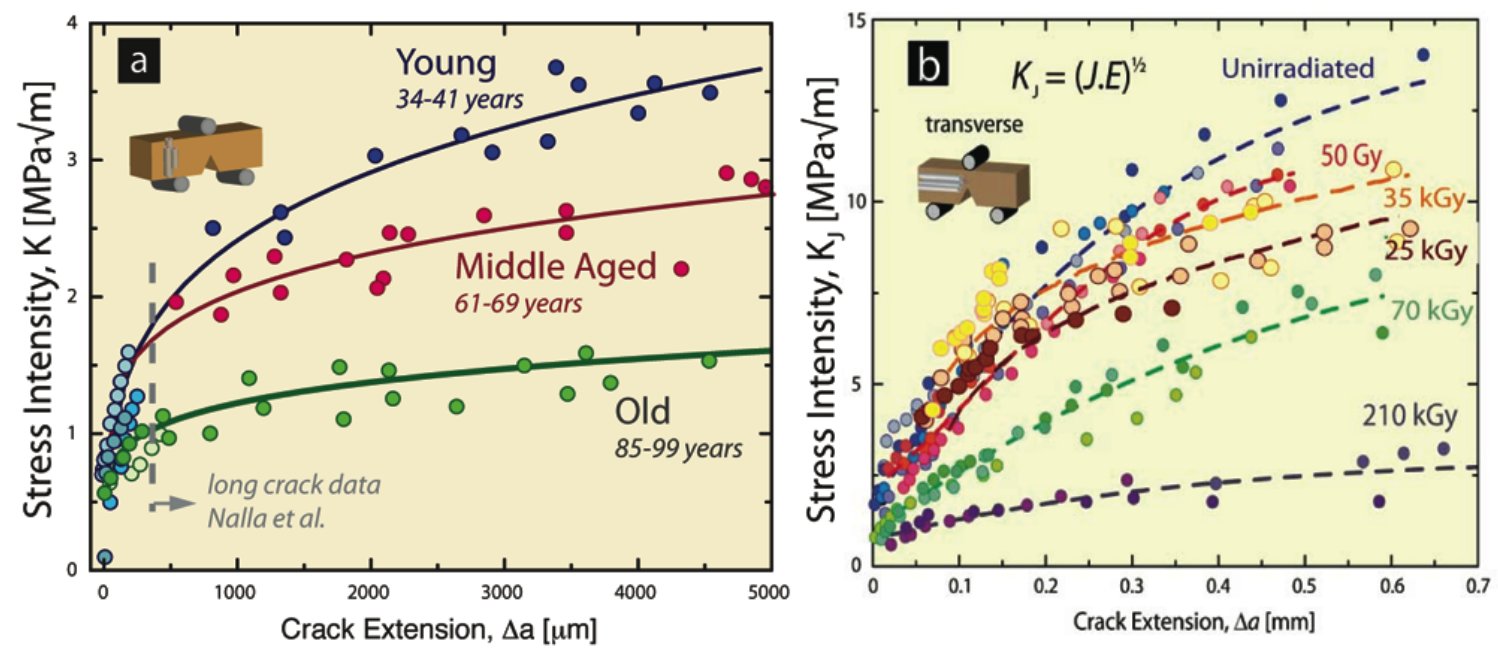

Fig. 2: As bone derives its toughness during crack initiation and growth, the toughness of human cortical bone is best characterized via its crack-growth resistance curve or R-curve, which demonstrates the crack initiation toughness as well as the evolution of the resistance to crack extension. Both (a) biological aging and (b) irradiation of bone result in a dramatic decrease in the crack initiation and growth toughnesses. Adapted from Zimmermann et al. and Barth et al. $(5,10)$. 


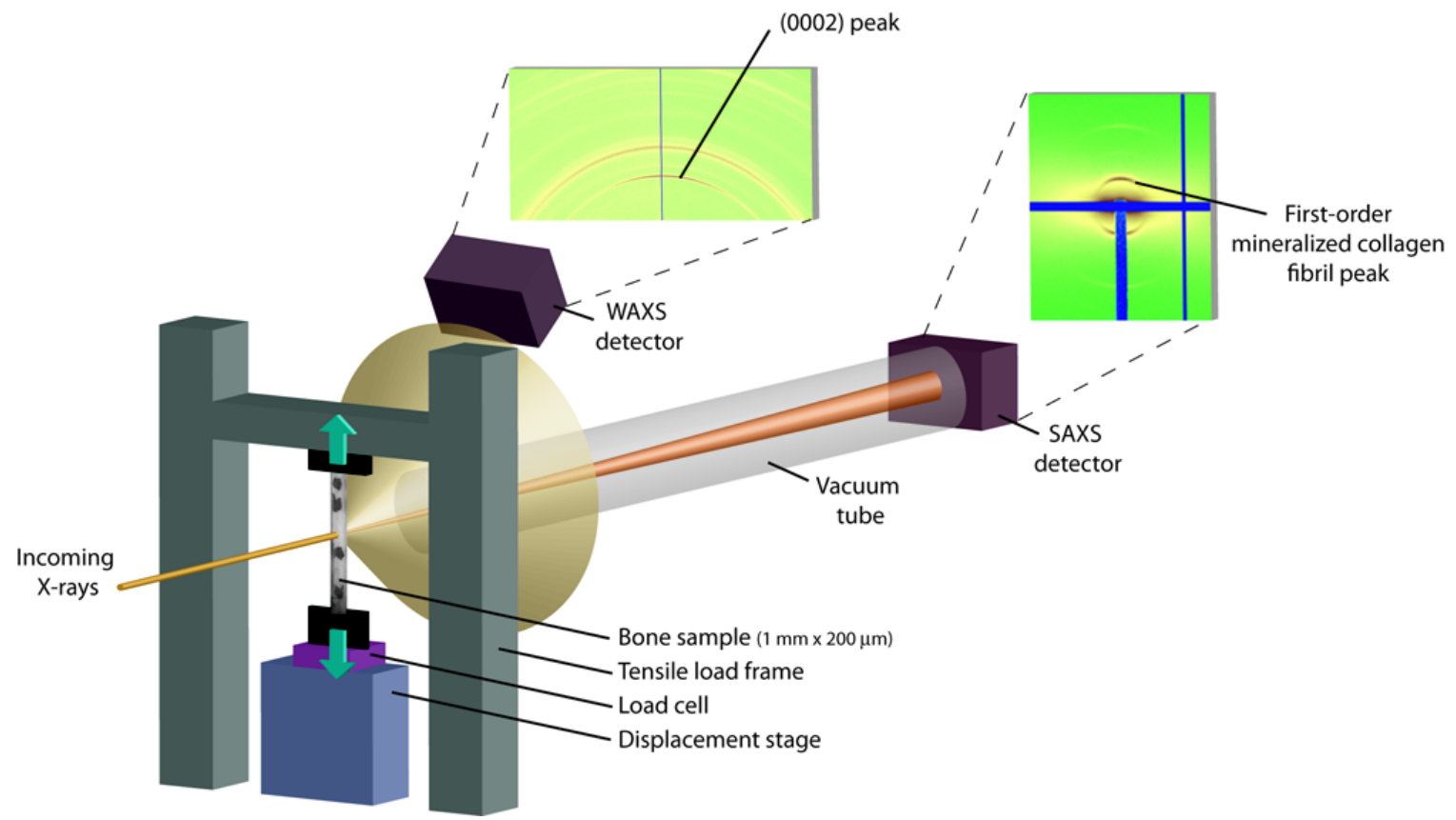

Fig. 3: For aging and radiation studies to examine the intrinsic (plasticity) mechanisms at submicron length-scales $(5,10)$, tension tests were performed in the x-ray synchrotron with a custombuilt tension rig that applied displacement to the bone tissue sample. Using a procedure similar to that used by Gupta et al. (25), the bone samples were exposed to high flux x-ray radiation causing the 67-nm stagger in the mineralized collagen fibril to scatter x-rays at a small angle (i.e., SAXS) and the lattice of the hydroxyapatite crystals to diffract x-rays at a large angle (i.e., WAXD). For a specific load applied to the bone sample, changes in the peak positions were used to calculate the individual strains in the mineral and fibrils strains, while the tissue strain was measured with a CCD camera (not pictured). 

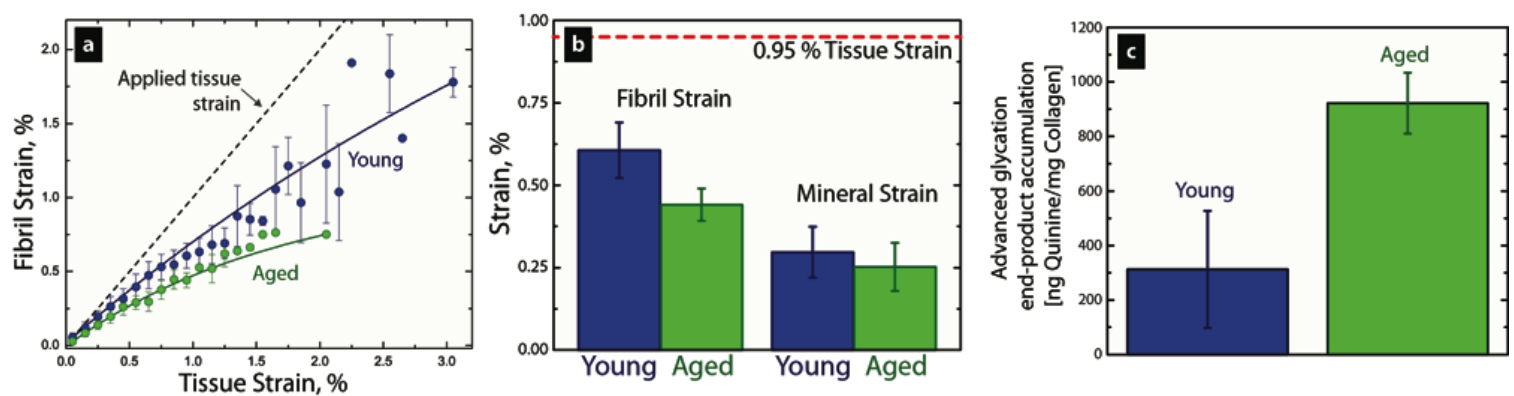

Fig. 4: Results of the SAXS/WAXD analyses with simultaneous mechanical testing (Fig. 3) were used to investigate the reduced plasticity exhibited by Aged bone in macroscopic mechanical testing (Fig. 2b). The SAXS/WAXD experiments $(a, b)$ demonstrate that for a given applied tissue strain, the strain in the Aged mineralized collagen fibril is $25 \%$ less than the Young fibril. (c) Consistent with constrained deformation in the Aged fibril, the Aged bone in this sample set contained three times as many AGEs cross-links, which strongly implies reduced fibrillar sliding within the Aged mineralized collagen fibril. Adapted from Zimmermann et al. (5). 

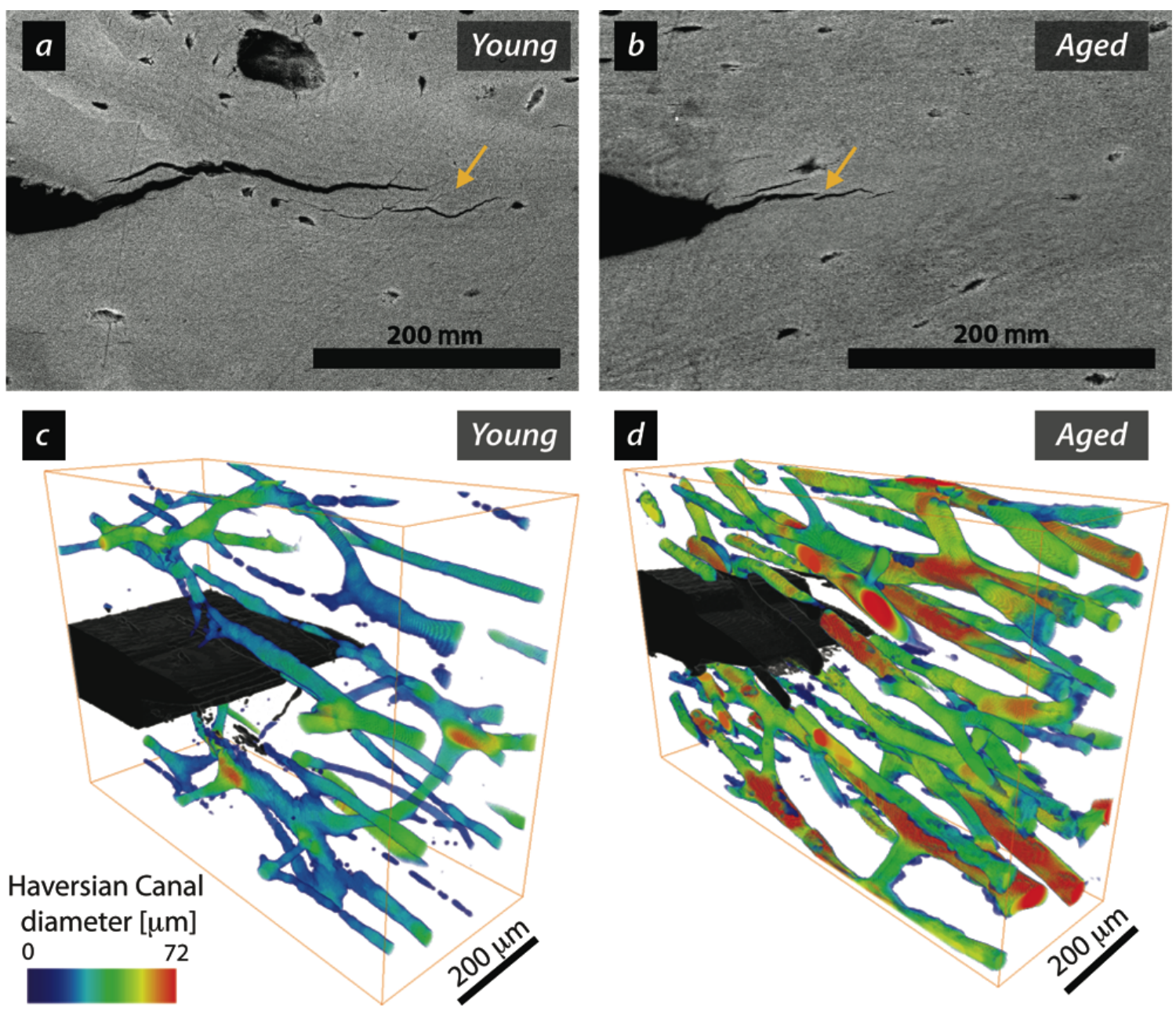

Fig. 5: The effect of biological aging on the extrinsic toughness can be investigated by imaging the crack path $(a, b)$ in 2-D with environmental scanning electron microscopy and $(c, d)$ in 3-D with synchrotron x-ray computed micro-tomography (tomographic images were taken and analyzed by Hrishikesh Bale). The crack-growth toughness of Aged human cortical bone is severely degraded by the limited uncracked ligament bridging; (a) in Young bone, the bridges form and shield the crack-tip from the full stress intensity, but (b) in Aged bone, the bridges are far smaller and occur less often. (c,d) The primary cause for the infrequency of crack bridges in Aged bone is the higher osteon density in comparison to Young bone, which is a result of higher turnover. Adapted from Zimmermann et al. (5). 

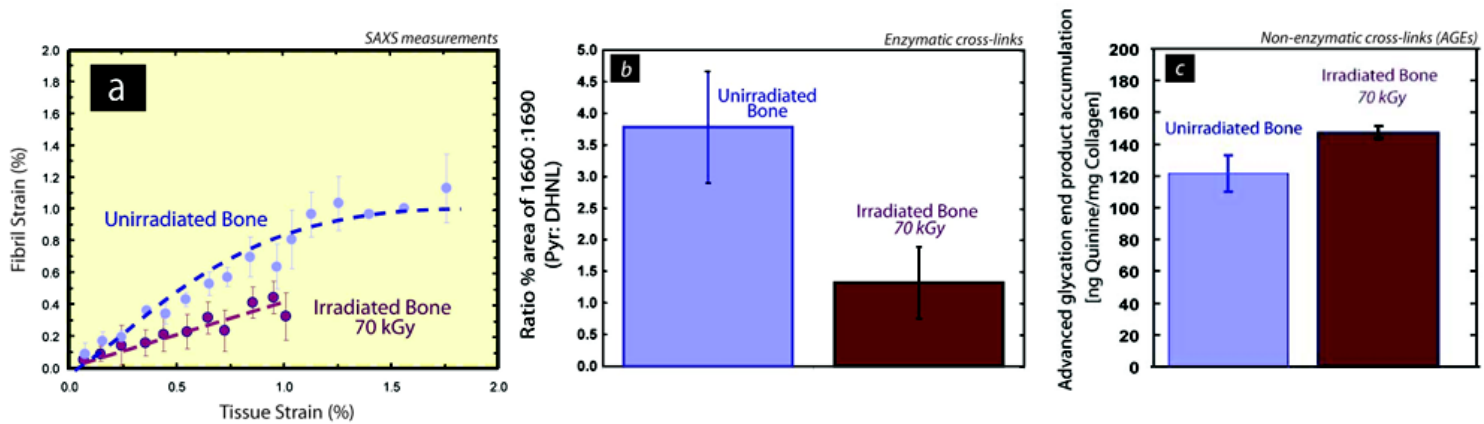

Fig. 6: Akin to aging, $x$-ray irradiation damage can also significantly limit bone's plasticity. Specifically, SAXS/WAXD experiments demonstrate (a) a substantial decreases in the ability of the mineralized collagen fibrils to plastically deform with irradiation. The main cause for the decrease in fibrillar sliding is the altered cross-linking profile, revealed as (b) a decrease in the ratio of mature to immature enzymatic cross-links and (c) an increase in non-enzymatic crosslinking. Adapted from Barth et al. (10). 
Non-irradiated bone

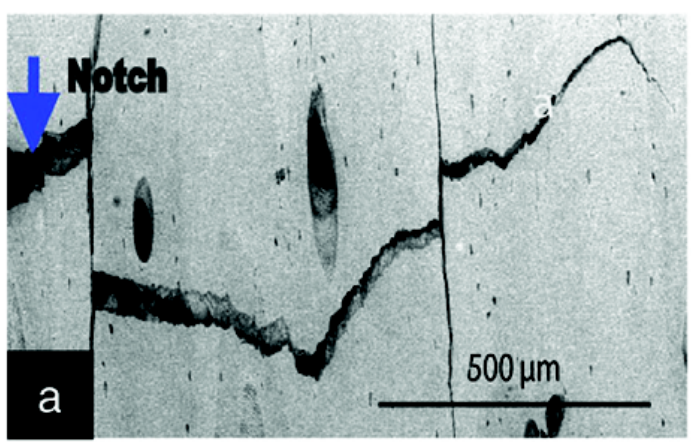

210 kGy irradiated bone

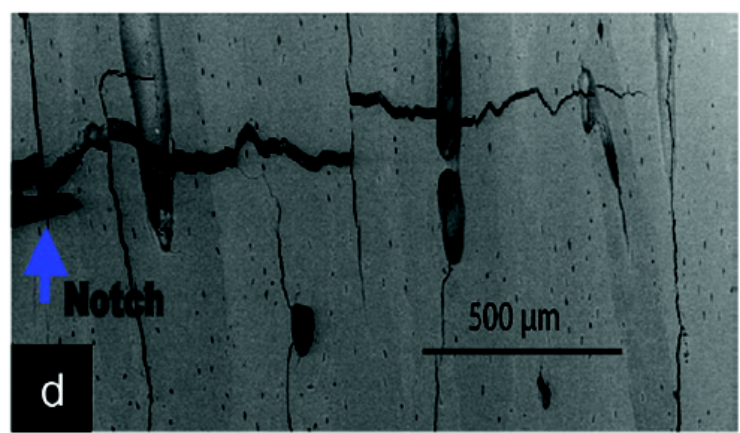

Fig. 7: The damage to the collagen matrix due to irradiation has an additional effect on the crackgrowth resistance in irradiated bone, as seen through the deterioration in the extrinsic crackgrowth toughness (i.e., the slope of the R-curve). The main driver for crack-growth toughness in (a) non-irradiated healthy bone is the extrinsic toughening mechanism of crack deflection, most notably as the crack encounters the osteonal interfaces (cement lines); (b) conversely, irradiated bone exhibits much smaller (although a greater number of) crack deflections, which actually leads to straighter, less tortuous, crack paths and a corresponding lower toughness. Adapted from Barth et al. (6). 